\title{
Periodic Solution of n-Species Gilpin-Ayala Competition System with Impulsive Perturbations
}

\author{
Kaihua Wang, Zhanji Gui* \\ School of Mathematics and Statistics, Hainan Normal University, Haikou, Hainan, China. \\ Email: *zhanjigui@sohu.com
}

Received 2012

\begin{abstract}
The principle aim of this paper is to explore the existence of periodic solution of n-Species Gilpin-Ayala competition system with impulsive perturbations. Sufficient and realistic conditions are obtained by using Mawhin's continuation theorem of the coincidence degree. Further, some numerical simulations show that our model can occur in many forms of complexities including periodic oscillation and chaotic strange attractor.
\end{abstract}

Keywords: Periodic Solution; Impulsive Perturbations; Mawhin’s Continuation Theorem

\section{Introduction}

The dynamics of Ayala-Gilpin competitive system, which was first introduced by Ayala et al. [1], has been widely studied by many authors [2-6]. However, the corresponding problems with periodic coefficients and impulsive perturbations were studied far less often [7]. In this paper, we will study the following impulsive Gilpin-Ayala system:

$$
\left\{\begin{aligned}
\dot{z}_{i}(t)= & r_{i}(t) z_{i}(t) \cdot \\
& {\left[1-\left(\frac{z_{i}(t)}{K_{i}(t)}\right)^{\theta_{i}}-\sum_{j=1, j \neq i}^{n} \alpha_{i j}(t) \frac{z_{j}(t)}{K_{j}(t)}\right], } \\
\Delta z_{i}\left(t_{k}\right) & =z_{i}\left(t_{k}^{+}\right)-z_{i}\left(t_{k}^{-}\right)=p_{k}^{i} z_{i}\left(t_{k}^{-}\right), t=t_{k},
\end{aligned}\right.
$$

where $z_{i}(t)$ represents the density of the $i$ th species at time $t ; r_{i}(t)$ denotes the intrinsic growth rate of the $i$ th species; $K_{i}(t)$ means the environment carrying capacity of species $i$ in the absence of competition; $\alpha_{i j}(t)(i \neq j)$ measures the amount of competition between the species $x_{i}$ and $x_{j} ; \theta_{i}$ is a positive constant and provide a nonlinear measure of intra-specific interference; $p_{k}^{i}$ are constants.

In system (1.1), we give two hypotheses as follows.

(H1) $r_{i}(t), K_{i}(t)$ and $\alpha_{i j}(t) \quad(i, j=1, \cdots, n, i \neq j)$ are all nonnegative $T$ - periodic functions defined on $R$.
(H2) $1+p_{k}^{i}>0$ and there exists a positive integer $q$ such that $t_{k+q}=t_{k}+T, p_{k+q}^{i}=p_{k}^{i}$.

\section{Existence of Positive Solutions}

To prove our results, we need the notion of the Mawhin's continuation theorem formulated in [8].

Lemma 1 ([8]) Let $X$ and $Y$ be two Banach spaces. Consider an operator equation $L X=\lambda N x$ where $L$ : Dom $L \cap X \rightarrow Y$ is a Fredholm operator of index zero and $\lambda \in[0,1]$ is a parameter, then there exist two projectors $P: X \rightarrow X$ and $Q: Y \rightarrow Y$ such that $\operatorname{Im} P=\operatorname{Ker} L$ and $\operatorname{Im} L=\operatorname{Ker} Q$. Assume that $N: \bar{\Omega} \rightarrow Y$ is $L$ compact on $\bar{\Omega}$, where $\Omega$ is open bounded in $X$. Furthermore, assume that

a) for each $\lambda \in(0,1), \quad x \in \partial \Omega \bigcap \operatorname{Dom} L, \quad L x \neq \lambda N x$;

b) for each $x \in \partial \Omega \bigcap \operatorname{Ker} L, Q N x \neq 0$;

c) $\operatorname{deg}\{J Q N, \Omega \cap$ Ker $L, 0\} \neq 0$, where $J: \operatorname{Im} Q \rightarrow$ Ker $L$ is an isomorphism and $\operatorname{deg}\{*\}$ represents the Brouwer degree.

Then the equation $L x=N x$ has at least one solution in $\bar{\Omega} \bigcap \operatorname{Dom} L$.

For the sake of convenience, we shall make some preparation. Let $I \subset R$. Denote by $P C\left(I, R^{n}\right)$ the space of functions $x(t): I \rightarrow R^{n}$ which are continuous at $t \in I, t \neq t_{k}$, and are left continuous for

*Corresponding author. 
$t=t_{k} \in I$. Let $u^{L}=\min _{0 \leq t \leq T}\{u(t)\}, u^{M}=\max _{0 \leq t \leq T}\{u(t)\}$, $\bar{u}=\frac{1}{T} \int_{0}^{T} u(t) d t, \overline{u v}=\frac{1}{T} \int_{0}^{T} u(t) v(t) d t$,

where $u(t), v(t)$ are $T$ periodic functions.

Theorem 1. Suppose (H1) and (H2) hold, furthermore, the following conditions are satisfied.

(H1) $\sum_{k=1}^{q} \ln \left(1+p_{k}^{i}\right)+\bar{r}_{i} T>\sum_{j=1, j \neq i}^{n} \frac{1}{K_{j}^{L}} \overline{r_{i} \alpha_{i j}} T e^{C_{j}}$,

where

$$
\begin{aligned}
C_{j}= & \frac{\ln \left[1+\frac{1}{\bar{r}_{j} T} \sum_{k=1}^{q} \ln \left(1+p_{k}^{j}\right)\right]+\ln K_{j}^{M}}{\theta_{j}}+2 \bar{r}_{j} T \\
& +\sum_{k=1}^{q} \ln \left(1+p_{k}^{j}\right)+\sum_{k=1}^{q}\left|\ln \left(1+p_{k}^{j}\right)\right| .
\end{aligned}
$$

Then system (1.1) has at least one positive $T$ - periodic solution.

Proof. Let

$$
z_{i}(t)=e^{x_{i}(t)} \quad(i=1, \cdots, n)
$$

then the system (1.1) becomes

$$
\left\{\begin{array}{l}
\dot{x}_{i}(t)=r_{i}(t)\left[1-\frac{e^{\theta_{i} x_{i}(t)}}{K_{i}^{\theta_{i}}(t)}-\sum_{j=1, j \neq i}^{n} \alpha_{i j}(t) \frac{e^{x_{j}(t)}}{K_{j}(t)}\right] t \neq t_{k} \\
\Delta x_{i}\left(t_{k}\right)=\ln \left(1+p_{k}^{i}\right) \quad t=t_{k}
\end{array}\right.
$$

In order to use Lemma 1, we set

$$
\begin{gathered}
X=\left(x_{1}(t), \cdots, x_{n}(t)\right)^{T}, \\
X=\left\{x \in P C\left(R, R^{n}\right) \mid x(t+T)=x(t)\right\}, \\
Y=X \times R^{n q},
\end{gathered}
$$

then it is standard to show that both $X$ and $Y$ are Banach space when they are endowed with the norm $\|x\|_{c}=\sup _{t \in[0, T]}|x(t)|$ and

$\left\|\left(x, c_{1}, \ldots, c_{q}\right)\right\|=\left(\|x\|_{c}^{2}+\left|c_{1}\right|^{2}+\cdots+\left|c_{q}\right|^{2}\right)^{1 / 2}$.

Set $L: \operatorname{Dom} L \subset X \rightarrow Y$ as

$$
(L x)(t)=\left(\dot{x}(t), \Delta x\left(t_{1}\right), \cdots, \Delta x\left(t_{q}\right)\right) \text {, }
$$

where $\operatorname{Dom} L=\left\{x \in X \mid x^{\prime}(t) \in P C\left(R, R^{n}\right)\right\}$,

$$
\operatorname{Im} L=\left\{\left(y, c_{1}, \cdots, c_{q}\right) \in Y \mid \int_{0}^{T} y(t) d t+\sum_{i=1}^{q} c_{i}=0\right\}
$$

and $\operatorname{Ker} \operatorname{Ker} L=R^{n}$.

At the same time, we denote $N: X \rightarrow Y$ as

$$
\begin{aligned}
& (N x)(t)=\left(f(t, x(t)), \Phi_{1}\left(x\left(t_{1}\right)\right), \cdots, \Phi_{q}\left(x\left(t_{q}\right)\right)\right), \\
& f(t, x) \\
& \text { where }=\left[r_{i}(t)\left(1-\frac{e^{\theta_{i} x_{i}(t)}}{K_{i}^{\theta_{i}}(t)}-\sum_{j=1, j \neq i}^{n} \alpha_{i j}(t) \frac{e^{x_{j}(t)}}{K_{j}(t)}\right)\right]_{n \times 1} \text {, } \\
& \Phi_{k}\left(x\left(t_{k}\right)\right)=\left(\ln \left(1+p_{k}^{1}\right), \cdots, \ln \left(1+p_{k}^{n}\right)\right)^{T},
\end{aligned}
$$

where $i=1, \cdots, n, k=1,2, \cdots, q$.

Define two projectors $P$ and $Q$ as

$P: X \rightarrow \operatorname{ker} L, P x=\frac{1}{T} \int_{0}^{T} x(t) d t ; Q: Y \rightarrow Y$,

$Q\left(y, c_{1}, \ldots, c_{q}\right)=\frac{1}{T}\left[\int_{0}^{T} y(s) d s+\sum_{k=1}^{q} c_{k}, 0, \cdots, 0\right]$.

It can be easily proved that $L$ is a Fredholm operator of index zero, that $P, Q$ are projectors, and that $N$ is $L$ - compact on $\bar{\Omega}$ for any given open and bound subset $\Omega$ in $X$.

Now we are in a position to search for an appropriate open bounded subset $\Omega$ for the application of Lemma 1 corresponding to operator equation

$$
L x=\lambda N x, \lambda \in(0,1)
$$

Suppose that $x(t)=\left(x_{1}(t), \cdots, x_{n}(t)\right)^{T}$ is a periodic solution of (2.3) for certain $\lambda \in(0,1)$. By integrating (2.3) over the interval $[0, T]$, we get

$$
\begin{aligned}
\overline{r_{i} T=} & -\sum_{k=1}^{q} \ln \left(1+p_{k}^{i}\right)+\int_{0}^{T} \frac{r_{i}(t)}{K_{i}^{\theta_{i}}(t)} e^{\theta_{i} x_{i}(t)} d t \\
& +\sum_{j=1, j \neq i}^{n} \int_{0}^{T} r_{i}(t) \alpha_{i j}(t) \frac{e^{x_{j}(t)}}{K_{j}(t)} d t
\end{aligned}
$$

From (2.3), (2.4), we can obtain

$\int_{0}^{T}\left|\dot{x}_{i}(t)\right| d t \leq 2 \bar{r}_{i} T+\sum_{k=1}^{q} \ln \left(1+p_{k}^{i}\right) \equiv A_{i}$

Since $\quad x_{i}(t) \in P C([0, T], R) \quad$, there exist $\xi_{i}, \eta_{i} \in[0, T] \bigcup\left\{t_{1}^{+}, t_{2}^{+}, \cdots, t_{q}^{+}\right\}$, such that

$$
x_{i}\left(\xi_{i}\right)=\inf _{t \in[0, T]} x_{i}(t), x_{i}\left(\eta_{i}\right)=\sup _{t \in[0, T]} x_{i}(t),
$$

It follows from (2.4) that

which implies 


$$
x_{i}\left(\xi_{i}\right) \leq \frac{\ln \left[1+\frac{1}{\bar{r}_{i} T} \sum_{k=1}^{q} \ln \left(1+p_{k}^{i}\right)\right]+\ln K_{i}^{M}}{\theta_{i}} \equiv B_{i}
$$

Thus we get

$$
\begin{aligned}
x_{i}(t) & \leq x_{i}\left(\xi_{i}\right)+\int_{0}^{T}\left|\dot{x}_{i}(t)\right| d \neq \sum_{k=1}^{q}\left|\ln \left(1+p_{k}^{i}\right)\right| \\
& \leq B_{i}+A_{i}+\sum_{k=1}^{q}\left|\ln \left(1+p_{k}^{i}\right)\right| \equiv C_{i}
\end{aligned}
$$

In particular, we have $x_{i}\left(\eta_{i}\right) \leq C_{i}$.

On the other hand, from (2.4), we have

$$
\begin{aligned}
\overline{r_{i} T \leq} & -\sum_{k=1}^{q} \ln \left(1+p_{k}^{i}\right)+\frac{\overline{r_{i} T}}{K_{i}^{L}} e^{\theta_{i} x_{i}\left(\eta_{i}\right)} \\
& +\sum_{j=1, j \neq i}^{n} \frac{1}{K_{j}^{L}} \overline{r_{i} \alpha_{i j}} T e^{x_{j}\left(\eta_{j}\right)}
\end{aligned} .
$$

Then we get

$$
\frac{\overline{r_{i} T}}{K_{i}^{L}} e^{\theta_{i} x_{i}\left(\eta_{i}\right)} \geq \sum_{k=1}^{q} \ln \left(1+p_{k}^{i}\right)+\overline{r_{i}} T-\sum_{j=1, j \neq i}^{n} \frac{1}{K_{j}^{L}} \overline{r_{i} \alpha_{i j}} T e^{C_{j}}
$$

Because of (H3) we have

$$
\begin{aligned}
& x_{i}\left(\eta_{i}\right) \geq \frac{\ln \left(K_{i}^{L}\right)-\ln \left(\overline{r_{i} T}\right)}{\theta_{i}}+ \\
& \frac{\ln \left(\sum_{k=1}^{q} \ln \left(1+p_{k}^{i}\right)+\overline{r_{i}} T-\sum_{j=1, j \neq i}^{n} \frac{1}{K_{j}^{L}} \overline{r_{i} \alpha_{i j}} T e^{C_{j}}\right)}{\theta_{i}} \equiv D_{i}
\end{aligned}
$$

Thus we get

$$
\begin{aligned}
x_{i}(t) & \geq x_{i}\left(\eta_{i}\right)-\int_{0}^{T}\left|\dot{x}_{i}(t)\right| d \neq \sum_{k=1}^{q}\left|\ln \left(1+p_{k}^{i}\right)\right| \\
& \geq D_{i}-A_{i}-\sum_{k=1}^{q}\left|\ln \left(1+p_{k}^{i}\right)\right| \equiv E_{i}
\end{aligned}
$$

From (2.6) and (2.7), it follows that

$$
\left|x_{i}(t)\right| \leq F_{i}=\max \left\{\left|C_{i}\right|,\left|E_{i}\right|\right\}
$$

Obviously, $F_{i}(i=1, \cdots, n)$ are independent of $\lambda$. Thus, there exists a constant $F>0$, such that $\max \left\{\left|x_{1}\right|, \cdots,\left|x_{n}\right|\right\} \leq F$. Let $r>F_{1}+\cdots+F_{n}+F$, $\Omega=\left\{x \in X:\|x\|_{c}<r\right\}$, then it is clear that $\Omega$ satisfies condition (a) of Lemma 1 and $N$ is $L$-compact on $\bar{\Omega}$.

when $x=\left(x_{1}, \cdots, x_{n}\right)^{T} \in \partial \Omega \bigcap \operatorname{Ker} L=\partial \Omega \bigcap R^{n}$, $x$ is a constant vector in $R^{n}$ with $\|x\|=r$. Thus
$Q N x \neq 0$.

Let $J: \operatorname{Im} Q \rightarrow \operatorname{Ker} L,(d, 0, \ldots, 0) \rightarrow d$. A direct computation gives $\operatorname{deg}\{J Q N, \Omega \bigcap$ ker $L, 0\} \neq 0$.

By now we have proved that $\Omega$ satisfies all the requirements in Mawhin's continuation theorem. Hence, (2.1) has at least one $T$ - periodic solution. By of (2.1), we derive that (1.1) has at least one positive $T$-periodic solution. The proof is complete.

\section{An Illustrative Example}

To easy to call functions, let $x_{i}(t)=z_{i}(t)$. In (1.1), we take $n=2, t_{k}=k T$,

$$
\begin{aligned}
r_{1}(t)=5+0.6 \sin t, & r_{2}(t)=4-0.4 \cos t, \\
K_{1}(t)=2+0.3 \sin t, & K_{2}(t)=2+0.1 \sin t, \\
\theta_{1}=1.5, & \theta_{2}=1.6, \\
\alpha_{12}(t)=0.8+0.1 \cos t, & \alpha_{21}(t)=0.9+0.2 \sin t .
\end{aligned}
$$

Obviously, $r_{1}(t), \quad r_{2}(t), K_{1}(t), \quad K_{2}(t), \alpha_{12}, \alpha_{21}$, satisfy (H1). $p_{k}^{1}=0.3, \quad p_{k}^{2}=0.2$.

If $T=\pi / 2$, then system (1.1) under the conditions (H5) has a unique $2 \pi$-periodic solution (In Figures 1-3, we take $\left.\left[x_{1}(0), x_{2}(0)\right]^{T}=[0.5,0.5]^{T}\right)$. Because of the influence of the period pulses, the influence of pulse is obvious.

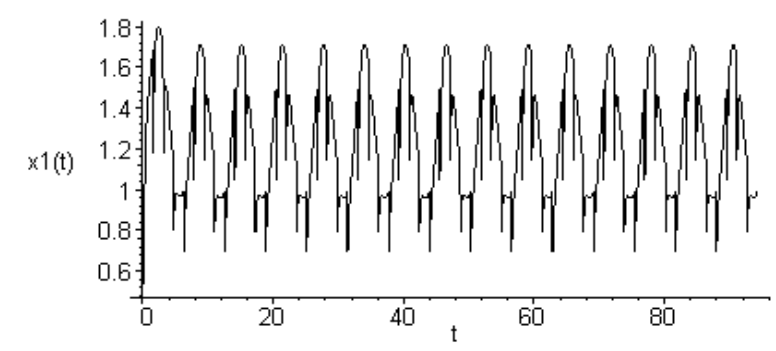

Figure 1. Time-series of $x_{1}(t)$ evolved in system (1) with $T=\pi / 2$.

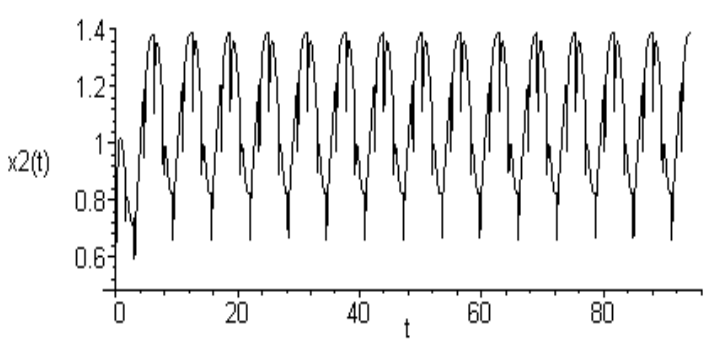

Figure 2. Time-series of $x_{2}(t)$ evolved in system (1) with $T=\pi / 2$. 
But if $T=2$, then (H2) is not satisfied. Periodic oscillation of system (1.1) under the conditions (H5) will be destroyed by impulsive effect. Numeric results show that system (1.1) under the conditions (H5) has gui chaotic strange attractor (see Figure 4) [9]. In Figure 4, we take $\left[x_{1}(0), x_{2}(0)\right]^{T}=[0.5,0.5]^{T}$.

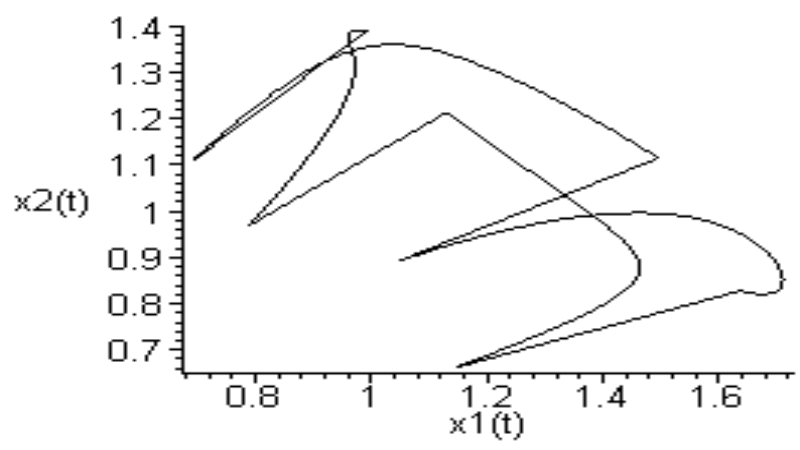

Figure 3. Phase portrait of periodic solutions of system (1) with $T=\pi / 2$.

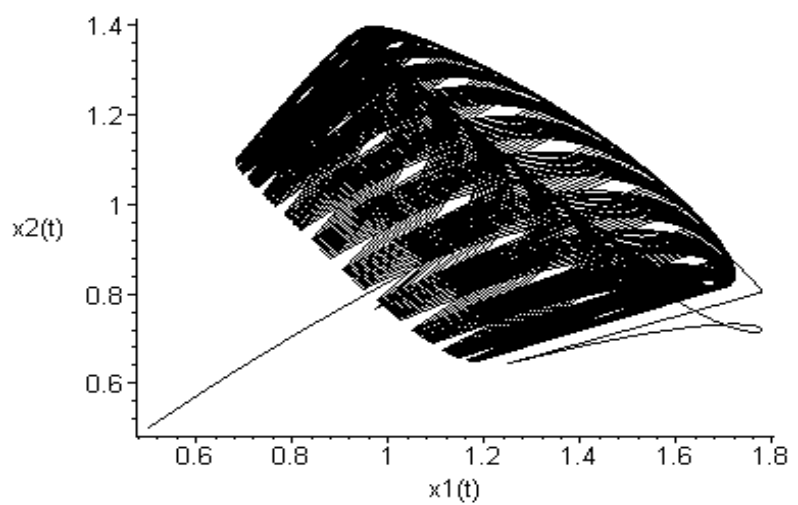

Figure 4. Phase portrait of chaotic strange attractor of system (1) with $T=2$.

\section{Acknowledgements}

This work is supported jointly by the Natural Sciences Foundation of China under Grant No. 60963025, Natural Sciences Foundation of Hainan Province under Grant No. 110007 and the Start-up fund of Hainan Normal University under Project No. 00203020201.

\section{REFERENCES}

[1] Ayala F J, Gilpin M E and Eherenfeld J G “Competition between species: Theoretical models and experimental tests,” Theoret. Popul. Biol. 4 (1973) 331-56.

[2] Gilpin M E and Ayala F J "Global models of growth and competition,” Proc. Natl. Acad. Sci. USA. 70 (1973) 3590-93.

[3] Liao X X and Li J "Stability in Gilpin-Ayala competition models with diffusion,” Nonlinear Anal. 28 19971751-58

[4] Ahmad S and Lazer A C “Average conditions for global asymptotic stability in a nonautonomous Lotka-Volterra system,” Nonlinear Anal. 40 (2000) 37-49.

[5] Chen F D "Average conditions for permanence and extinction in nonautonomous Gilpin-Ayala competition model,” Nonlinear Anal. 4 (2006) 895-915.

[6] Xia Y H, Han M A and Huang Z K “Global attractivity of an almost periodic N-Species nonlinear ecological competitive model,” J. Math. Anal. Appl. 337 (2008) 144-168.

[7] Wang Q, Ding M M, Wang Z J and Zhang H Y "Existence and attractivity of a periodic solution for an N-species Gilpin-Ayala impulsive competition system" Nonlinear Ana: Real World Applications 11 (2010) 2675-85

[8] Gaines R E and Mawhin J L "Coincidence degree and nonlinear diferential equations ” Springer-Verlag, Berlin, 1977.

[9] Zhang J and Gui Z J "Periodic solutions of nonautonomous cellular neural networks with impulses and delays," Nonlinear Analysis: Real World Applications. 10 (2009) 1891-1903. 